\title{
Universo da Psicologia: Serious Game para Promoção do Aprendizado no Sertão Baiano
}

\author{
Dandara B. Palhano ${ }^{1}$, Amanda C. A. de Oliveira ${ }^{1}$, Arkellenise M. N. B. Leite', \\ Brenda D. S. de Araújo ${ }^{1}$, Marcos A. M. da Silva ${ }^{1}$, Maria Clara Sandes ${ }^{1}$, Maynara \\ S. de Araújo ${ }^{1}$, Pedro A. L. Vilela ${ }^{1}$, Victor F. A. dos Santos ${ }^{1}$, Nickolas R. dos S. e \\ Santos ${ }^{1}$, João N. dos S. Neto'. \\ ${ }^{1}$ Bacharelado em Psicologia - Centro Universitário do Rio São Francisco (UNIRIOS) - \\ 48.603-004 - Paulo Afonso - BA - Brasil. \\ \{palhanodandara, amandaalexandre780, arkellenisemaria,mclarasandesz, mayn \\ arasdearaujo, peulima14\} @gmail.com, \\ \{brenda.daniely, marcos.a.moreir4,joaonanuca_petro\}@outlook.com, \\ \{victorchavier, nickolasleosantos\} @hotmail.com
}

\begin{abstract}
Scholar evasion of higher education reaches the most diverse layers, especially outside capital cities and less developed areas. Serious Games (SG) are a valid alternative in this context, combining playfulness with the specific purpose of learning. This article presents the $S G$ 'Universe of Psychology' whose design was based on the expanded tetrad aimed at specific content and which makes use of an intelligence system based on the Item Response Theory (IRT).
\end{abstract}

Resumo. A evasão do ensino superior atinge as mais diversas camadas, em especial no sertão. Os Serious Games (SG) são uma alternativa válida neste contexto, aliando o lúdico com o propósito específico do aprendizado. Apresenta-se neste artigo o SG Universo da Psicologia cujo design baseouse na tétrade expandida voltada ao conteúdo especifico e que faz uso de um sistema de inteligência baseado na Teoria de Resposta ao Item (TRI).

\section{Introdução}

SG são aplicações voltadas a um propósito. Este tipo de jogo é cuidadosamente pensado para atingir um objetivo, educativo, por exemplo, contudo, apesar de conter os aspectos do entretenimento e da diversão, este não é o primeiro, nem o principal propósito [Michael, David e Chen 2005]. Em geral os SG utilizam os mesmos recursos dos jogos casuais no que concerne a arte das aplicações gráficas para passar uma mensagem, promover o aprendizado ou favorecer a ocorrência de uma experiência. Portanto, SG incluem conteúdos para estimular o aprendizado, entre outros objetivos. Ratan, Radinbra e Ritterfeld (2009) justificam o uso destes jogos por eles agirem potencializando o processo de aprendizagem, tendo em vista que jogos interativos produzem mais efeito para a aprendizagem do que a pedagogia tradicional.

Para que um SG possa atingir seu objetivo, é necessário que o processo de planejamento e elaboração seja minucioso, determinando etapas específicas do desenvolvimento que agrupem os recursos lúdicos e o conteúdo específico. Diante disso, é necessário que uma equipe multidisciplinar esteja composta em sua construção, 
tendo em vista que diversas áreas do conhecimento trabalham de forma interdisciplinar com a indústria dos SG [Machado, Costa e Moraes 2018].

Desde a origem do ensino superior no Brasil, este é reservado a uma pequena parcela da população, devido ao seu alto grau de seletividade no acesso. Nos anos 90 houve ampla expansão de vagas, especialmente no setor privado, concentrando-se em áreas metropolitanas [Neves, Raizer e Fachinetto 2007]. Contudo a expansão do ensino superior chegou também às áreas mais afastadas das capitais, conseguindo adentrar a outras camadas da população, sobretudo ampliando o acesso a negros, indígenas e oriundos de escolas públicas. De acordo com Santos e Silva (2011) o principal problema refere-se ao fato de que inexistiam, à época, programas que visassem apoiar esses segmentos, possibilitando a ampliação da evasão escolar. Apesar disso, os autores também afirmam que o abandono do ensino superior não é restrito à classe popular, pois é comum que o estudante se sinta inseguro ao entrar na vida universitária, devido à sua forma diferenciada de ensino-aprendizado. Em pesquisa recente, encontrou-se dados que mostram que a evasão no ensino superior no Brasil atingiu $30,1 \%$ na rede privada e 18,5\% na rede pública [Bocchini 2018].

O objetivo deste trabalho é apresentar o desenvolvimento de um SG visando à promoção de aprendizado para o curso de Bacharelado em Psicologia, usando um sistema de inteligência baseado na Teoria de Resposta ao Item (TRI). A TRI é amplamente utilizada para avaliação educacional, tendo como principais exemplos sua aplicação nos Exames Nacionais do Ensino Médio (ENEM) e do Desempenho de Estudantes (ENADE) [Brasil 2011].

Em pesquisa realizada pelos autores, não foram encontradas alternativas de aprendizagem como o uso de SG, por exemplo, para o curso de Psicologia. Parte da motivação para o desenvolvimento deste SG vem do cenário de estudo do curso de Psicologia no sertão baiano, que contempla variáveis promotoras da evasão escolar, tais como, estudantes oriundos de escolas públicas, negros, indígenas e inseridos em contexto de vulnerabilidade social. Apesar desse cenário, alternativas precisam ser realizadas visando a redução do impacto de algumas destas variáveis preditoras da evasão. Uma destas variáveis diz respeito a dificuldades de aprendizagem ao lidar com um conteúdo novo e diferenciado do que estavam acostumados.

Com relação ao cenário pedagógico, os SG tornam-se espaços promotores de aprendizagem e abrem caminhos para diferentes estilos cognitivos e emocionais [Turkle 1997]. De acordo com Silva e Falcão (2019), essas tecnologias estão cada vez mais sendo inseridas no processo de aprendizagem de escolas e alunos por meio de dispositivos digitais ou projetos relacionados. Assim, a mediação da aprendizagem através de um SG pode promover dinâmica e interação necessárias para desafiar cognitivamente os aprendentes. A intenção não deve ser simplesmente animar o fazer pedagógico, mas sim motivar os alunos na interação com os objetos de conhecimento, podendo desenvolver conceitos distintos e articular a teoria com a prática.

\section{Método e Material}

O objetivo deste trabalho é apresentar o desenvolvimento de um SG para promoção do aprendizado no bacharelado em Psicologia, usando um sistema de inteligência baseado na TRI. Para este fim foram realizados estudos teóricos sobre metodologias de aprendizagem e das áreas e disciplinas do Bacharelado em Psicologia. 
Destaca-se que trabalho faz parte de um projeto de extensão denominado Psicologia Digital, voltado à inclusão de estudantes do Bacharelado em Psicologia no mundo digital dos SG, além de visar a promoção de aprendizado por meios tecnológicos modernos.

As atividades estão sendo modeladas considerando elementos de jogos baseados na tétrade expandida proposta por Machado, Costa e Moraes (2018), que sugere que a mecânica, a estética, a estória, e a tecnologia são permeadas pelo conteúdo específico do SG. Além disso, o SG é composto de regras e desafios com o objetivo de alcançar seu propósito, contudo, sem perder o caráter lúdico.

Os requerimentos, também propostos pelos autores [Machado, Costa e Moraes 2018] orientam algumas definições, assim definiu-se o público alvo como sujeitos estudantes do bacharelado em Psicologia, com idade entre 16 e 60 anos de todos os gêneros, que conheçam e usem computador e/ou celular.

O SG apresenta objetivos educacionais a partir da promoção de entretenimento e diversão. Busca-se gerar uma experiência lúdica a ser atingida a partir da mecânica, estética, história do SG e tecnologia [Barbosa e Murarolli 2013]. A história do SG está voltada às áreas da Psicologia, apresentadas em disciplinas tais como: processos epistemológicos, neuroanatomia, psicologia do desenvolvimento, processos psicológicos básicos, psicologia social, entre outras, visando assim, promover diversão e aprendizado.

Para a produção do SG é utilizada a engine Unity (www.unity.com), na modalidade 2D. Esta engine foi escolhida pela facilidade no desenvolvimento, por contar com uma loja de assets própria, vários fóruns de discussão resolutivos e por ser possível exportar para mais de 20 plataformas. Além disso, por meio da Unity é possível desenvolver jogos ou aplicativos em formato $2 \mathrm{D}$ ou $3 \mathrm{D}$, promovendo mais um motivo para a sua escolha, pela versatilidade. Esta engine utiliza a linguagem C\# para programação, sendo esta uma linguagem voltada ao objeto e, portanto, mais próxima para desenvolvedores principiantes que se iniciaram no mundo da programação por meio da linguagem $\mathrm{C}$ ou $\mathrm{C}++$.

Este SG é produzido para ocorrer em um espaço 2D, cuja estética é baseada em flat design permitindo ao SG a apresentação de características clean, sem muitos efeitos, sendo o design simples, porém moderno e priorizando mais as informações, elemento esse importante no SG, pois permite uma relação mais diretiva com o jogador [Barros Júnior 2016].

No Universo da Psicologia, a utilização da TRI está presente no processo de determinar o próximo desafio, próxima questão, que será apresentado ao jogador. Além de avaliar o entendimento do jogador sobre o assunto abordado no desafio, será uma nova maneira de determinar o quão capacitado o jogador está dentro do universo do jogo. Sendo assim a TRI é utilizada como sistema de inteligência e modelo de decisão. A TRI é um baseada em modelos estatísticos utilizados para cálculo do traço latente, também chamado de aptidão ou de construto. Esta teoria é eventualmente utilizada para a formação de instrumentos para conhecimento e comportamento [INEP 2012]. Deste modo, a TRI avalia item por item, em um grupo de itens válidos permitindo uma combinação de testes diferenciados conforme a resposta do jogador. 
O SG ocorre em níveis de complexidade, conforme apontado pela TRI. Sua história faz com que o jogador viaje pelo Universo da Psicologia, passando por planetas e estações espaciais referentes às áreas da Psicologia e suas disciplinas. O propósito é gerar diversos benefícios, bem como desenvolvimento de competências e aumento de produtividade.

\section{Desenvolvimento do Universo da Psicologia}

O processo de design de um SG demanda a participação de uma equipe multidisciplinar e precisa observar vários requerimentos. $\mathrm{O}$ design deste $\mathrm{SG}$ está sendo baseado na tétrade expandida proposta por Machado, Costa e Moraes (2018), que pode ser vista na figura 1. Destaca-se que esta tétrade é baseada na tétrade elementar de Schell (2011). A base necessária para o design do SG considera que o conteúdo específico permeia a mecânica, a estória, a estética e a tecnologia.

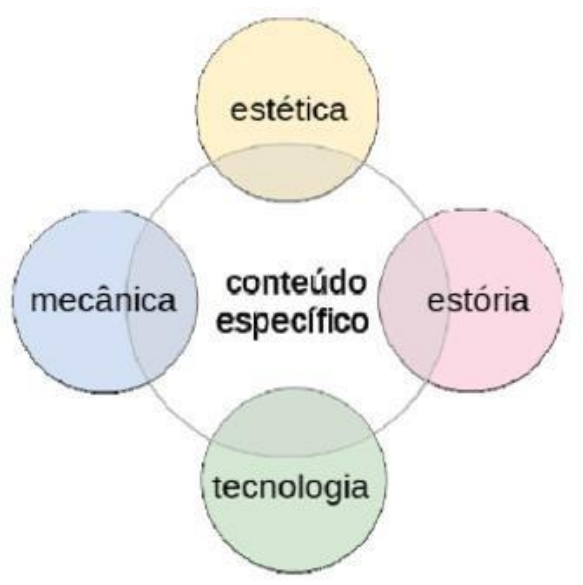

Figura 1. Tétrade Expandida com foco no conteúdo específico [Machado, Almeida e Moraes 2018].

\subsection{Conteúdo}

O conteúdo específico deste SG baseia-se em disciplinas do Bacharelado em Psicologia. As primeiras matérias escolhidas, foram Processos Epistemológicos, Neuroanatomia, Psicologia do Desenvolvimento e Processos Psicológicos Básicos, todas disciplinas iniciais do bacharelado.

$\mathrm{Na}$ disciplina de Processos Epistemológicos é estudada e revisada toda a história da Psicologia até os dias atuais, sendo um pontapé inicial para o curso e a profissão. Segundo Tesser (1994) a tarefa principal da epistemologia consiste na reconstrução racional do conhecimento científico, conhecer, analisar, todo o processo gnosiológico da ciência do ponto de vista lógico, linguístico, sociológico, interdisciplinar, político, filosófico e histórico.

Outra disciplina considerada é Neuroanatomia, considerando-se que a partir de processos cerebrais, estruturais ou de processamento, é possível apresentar distúrbios psíquicos, cognitivos, de personalidade, entre outros.

A Psicologia do Desenvolvimento, tem sua notoriedade dada pelo estudo das mudanças, sejam estas físicas, cognitivas ou psicossociais que o ser humano passa no decorrer da vida, dizendo muito sobre sua conjuntura. 
Por fim, a disciplina de Processos Psicológicos Básicos é a última disciplina base utilizada na primeira versão do SG, na qual assuntos recorrentes como a cognição, atenção e percepção, memória, linguagem, emoções, inteligência e motivação são estudados e considerados primordiais para a formação de profissionais na área

\subsection{Narrativa}

Narrativa é a sequência dos eventos que se desdobram no jogo [Machado, Costa e Moraes 2018][Schell 2011]. No caso de um SG ela tem relação direta com a estória despertando a diligência dos que desfrutam do mesmo. Tendo em vista a proposta de um SG voltado para a promoção do aprendizado em estudantes do Bacharelado em Psicologia, o SG foi proposto em sua primeira versão, a se passar em um universo onde os planetas iniciais são disciplinas básicas do curso, sendo assim destrinchado todo o Universo da Psicologia. específico:

Os planetas referem-se às disciplinas apresentadas no tópico do conteúdo

-Processos Epistemológicos: Planeta Epistemio;

-Neuroanatomia: Planeta Neuroanat;

-Psicologia do Desenvolvimento: Planeta Desenv; e

-Processos Psicológicos Básicos: Planeta PPB.

Além da escolha dos planetas em que o jogador pode aterrissar, ele pode também escolher seu personagem, considerando-se serão incluídas técnicas de engajamento relacionadas aos avatares selecionados.

\subsection{Mecânica}

A mecânica define o SG e como ele vai acontecer [Machado, Costa e Moraes 2018][Schell 2011] Quando um jogador entra em contato com um novo SG surgem indagações comuns sobre o funcionamento dele, sobre o que é necessário fazer e qual é o seu objetivo, então, os elementos da mecânica do SG trabalham nessas questões. De acordo com Machado, Costa e Moraes (2018) os SG quando gerados com um objetivo específico, para além do entretenimento, procuram exercitar as competências dos seus usuários e, por isso, é de grande relevância discriminar qual é o propósito específico dessas aplicações, definindo como abordar a problemática durante $o$ desenvolvimento, para que, desta forma, sua mecânica seja pensada visando o fomento do propósito do SG.

Podem-se incluir na mecânica do SG desafios, customizações, regras, rankings e recompensas como medalhas, pontuações e presentes. Para o desenvolvimento da mecânica é necessário considerar o engajamento que pode ser entendido como uma qualidade de experiência do usuário, sendo este caracterizado por atributos de desafio, afeto positivo, capacidade de execução, atrativo estético e sensorial, atenção, feedback, variedade / novidade, interatividade e controle de usuário percebido.

O processo de engajamento é composto por quatro estágios distintos, que adaptados ao contexto de SG podem ser entendidos como: Início do engajamento Refere-se a como o engajamento começa e qual o ponto de partida para começar a jogar; Período de engajamento sustentado: Refere-se ao que acontece e o que se sente e 
pensa enquanto se está envolvido; Desengajamento: ele ocorre quando, por uma decisão própria do jogador, ou por conta de fatores externos, há a interrupção do interesse de jogar; Reengajamento: Apesar de haver o desengajamento, este não configura uma perda total do interesse no jogo, visto que pode ocorrer reengajamento tanto em curto como em longo prazo [O’Brien e Toms 2008].

Pode haver também o não engajamento, ou seja, o sujeito não se engaja por fatores diversos [O’Brien ae Toms 2008]. É possível lançar mão da utilização de algumas técnicas de engajamento como as apresentadas por Sena e Coelho (2012). Estas técnicas podem ser usadas para incutir no jogador emoções e sentimentos que garantam seu interesse no SG. Pretende-se empregar neste SG algumas técnicas de engajamento, sendo elas prêmios virtuais que segundo Zichermann e Cunningham (2011) são uma maneira de apresentar as conclusões de metas e marcar o progresso no sistema do SG; segundo os mesmos autores desafios e missões, são essenciais para manter o jogador ocupado e evitar a sensação de fim do SG; outra técnica que pretende ser aplicada é o sistema de presentes no jogo, visando a interação social, estimulando o jogador a presentear seus amigos.

O SG possuirá níveis e um sistema de busca de questões através de palavraschave como autor ou obra, com a finalidade de proporcionar ao jogador a opção de uma aprendizagem exploratória, construtivista e direcionada a determinado autor ou determinada obra. Mattar (2010) propõe que a liberdade para experimentar, fornece aos alunos um poder de controle sobre o seu próprio aprendizado e atende a necessidade do nativo digital de explorar e se auto educar.

\subsection{Tecnologia}

Nos jogos há um processo de tomada de decisão da máquina, que ocorre de forma particular e específica através de um Sistema de Inteligência no jogo. Neste SG será utilizada como base do sistema de inteligência a TRI.

A TRI é uma teoria do traço latente, inicialmente aplicada a testes de habilidade ou desempenho [Pasquali 2007]. Estudiosos assinalam que a TRI pode ser descrita como um grupo de modelos psicométricos que busca desenvolver e refinar instrumentos psicológicos [Embretson e Reise 2000]. TRI refere-se a um conjunto de modelos matemáticos, a partir do qual se entende que a escolha de um sujeito depende do nível de traço latente que ele possui. Assim, a TRI avalia item a item resultando, em um grupo de itens válidos permitindo a elaboração de inúmeros testes diferenciados conforme as escolhas dos sujeitos ocorram.

A TRI traz dois postulados básicos, sendo eles: 1) a performance de um sujeito na tarefa pode ser predito ao se considerar um conjunto de variáveis hipotéticas, que são os traços latentes, identificados pela letra grega teta $(\theta)$; e 2) a relação entre o desempenho na tarefa e o traço latente e descrita por uma função matemática chamada Curva Característica do Item (CCI). A CCI aponta que na mesma medida que o nível de traço latente do sujeito aumenta, ou seja, o $\theta$ aumenta, aumenta a probabilidade de o sujeito escolher uma opção diante de uma tarefa. Dessa forma, pode-se compreender que o $\theta$ permite a estimação da resposta do indivíduo para cada tarefa, além de possibilitar um cálculo individual [Pasquali 2007].

$\mathrm{Na}$ TRI, existem três parâmetros que podem ser observados na CCI, contudo, a quantidade de parâmetros a ser observada depende do modelo adotado, podendo ser 
um, dois ou três parâmetros (dificuldade, discriminação e/ou chute) [Pasquali 2007]. A CCI consiste em expressar a probabilidade de um sujeito escolher uma resposta em função de seu traço latente. Assim, faz-se a pergunta sobre qual probabilidade o sujeito tem de acertar o item, dependendo esta, do nível de aptidão do sujeito. O modelo escolhido neste estudo é o de três parâmetros proposto por Birmbaum (1957) que coloca que a probabilidade do sujeito acertar o item está associada com a dificuldade, discriminação e o acerto ao acaso (chute). A utilização desta teoria dar-se-á neste estudo para a construção do sistema de inteligência para o SG, possibilitando também a verificação dos parâmetros das escolhas a serem feitas na aplicação.

\subsection{Estética}

Seguindo a tétrade elementar, chega-se à construção da estética do SG, um elemento extremamente importante no design de SGs, pois se relaciona de forma mais direta com o jogador [Schell 2011]. Levando em consideração os requisitos apontados por Machado, Costa e Moraes (2018), o SG será produzido para computadores e smartphones, portanto foram produzidas imagens em flat design.

Segundo Cristofolini e Oliari (2015) é possível classificar o flat design, também chamado de design plano, como uma estética visual de interface, sua proposta é descartar tudo que seja desnecessário visualmente, sejam sombras, drop shadows, relevos, gradientes ou texturas, assim, este tipo de design é composto de layouts limpos e com cores sólidas. Apesar da simplicidade das imagens, as cores são mais trabalhadas, de forma a fornecer uma experiência agradável ao jogador. Os autores acrescentam que para trabalhar com o estilo é importante saber usar bem as cores, conhecendo seus significados e adequar a comunicação de acordo com a identidade do público a ser abordado. Pode-se verificar na Figura 2 a primeira imagem com a qual o jogador se depara no SG Universo da Psicologia.

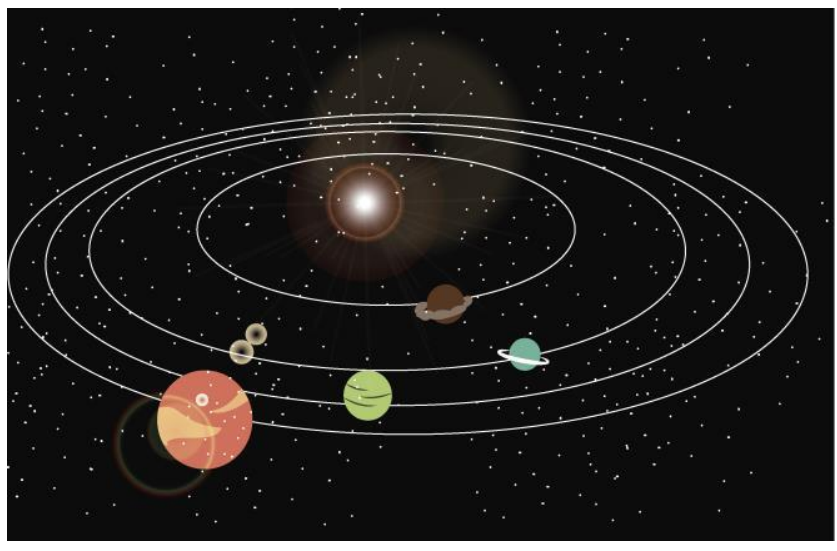

Figura 2. Abertura do Universo da Psicologia.

Os personagens foram pensados e realizados partindo de uma mistura de cartum e personagens de animes, mas com características regionais, destaca-se que eles poderão ser personalizados pelo jogador. O estilo foi escolhido a partir de uma perspetiva futurística, o que pode ser notado nos uniformes, com o objetivo de agradar diferentes públicos e ainda, dar aos personagens características nacionais. Na Figura 3 são apontados exemplos de personagens que podem ser escolhidos. 


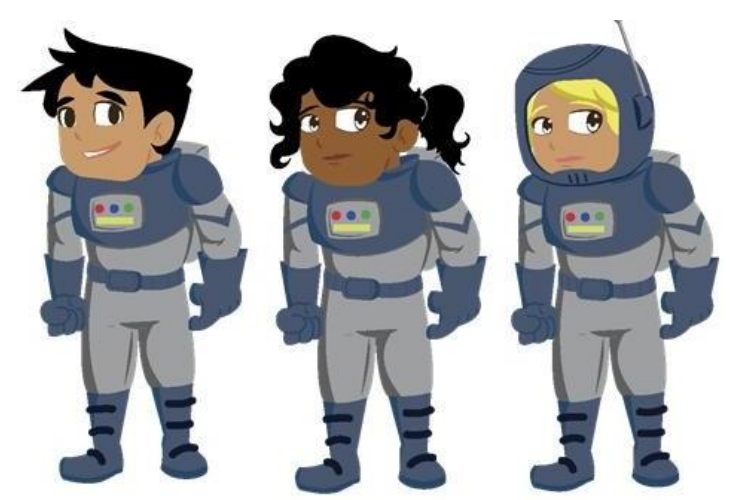

Figura 3. Personagens do Universo da Psicologia.

Imagens de dois planetas para os quais estão sendo desenvolvidas questões podem ser vistas na Figura 4. Como apontado no tópico referente à narrativa do SG, são o Planeta "Desenv", da Psicologia do Desenvolvimento e o Planeta "Epistemio" referente à Epistemiologia. As paisagens atribuídas a cada disciplina são pensadas de acordo com o conteúdo da disciplina, por exemplo, matérias mais iniciais do curso recebem paisagens de planetas em evolução, como o planeta "Epistemio" que aborda conteúdos históricos do início da psicologia, de forma a ganhar um ambiente rudimentar, o planeta "Desenv" já se encontra uma paisagem com mais desenvolvimento, conforme o nome da disciplina.

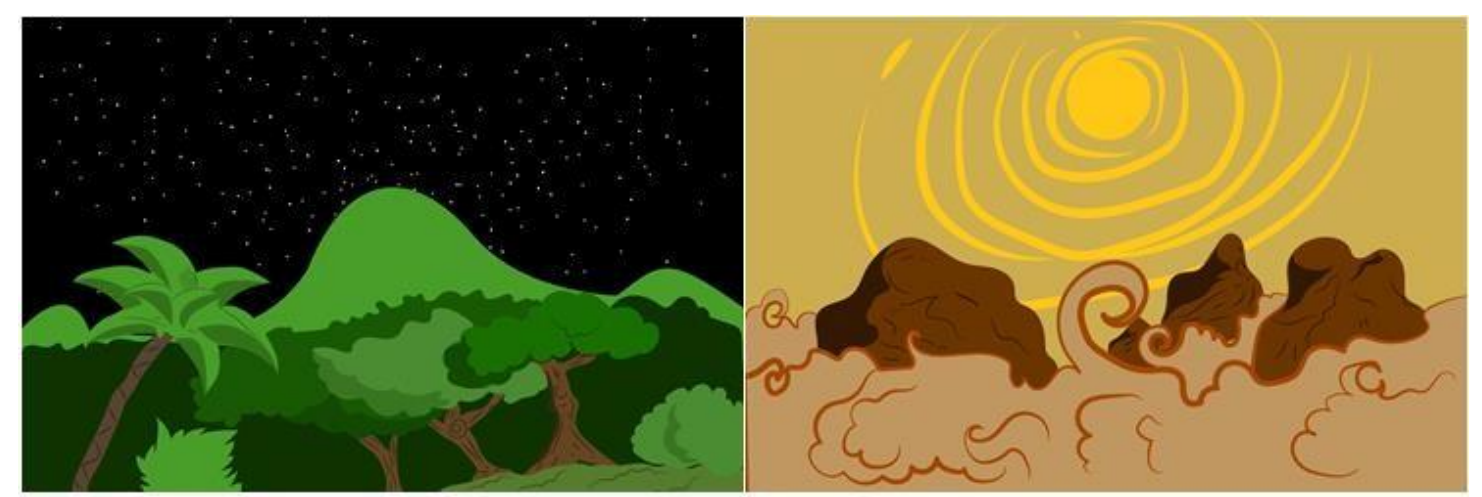

Figura 4. Planeta Desenv e Planeta Epistemio do Universo da Psicologia.

\section{Resultados}

O projeto conseguiu definir sua identidade visual, assim como uma mecânica que proporcione aprendizado e diversão, através de um conjunto de regras, sistema de recompensa e personalizações que visam uma maior imersão do jogador. A definição da narrativa conseguiu mesclar o conteúdo abordado na psicologia com uma viagem por um universo fictício, o que permite futuras atualizações que adicionem mais fases, objetivos e novos elementos. Um dos desafios na produção do SG é a implementação da TRI que proporcionará um diferencial ao jogo e uma experiência única para cada jogador.

Espera-se com este projeto alcançar o fomento à aprendizagem no bacharelado em Psicologia, em especial no sertão baiano, minimizando o potencial da variável 
relacionada à dificuldade de aprendizagem no ensino superior e diretamente ligada à possível evasão de estudantes do Ensino Superior. Entende-se que a cultura digital tem um alto potencial para transformar os ambientes de ensino em locais de aprendizagem ativo, possibilitando aos estudantes construir o aprendizado, ao invés de simplesmente estudar e decorar acumulando conhecimento [Valente e Martins 2011].

\section{Referências}

Barbosa, P. A., Murarolli, P. L. (2013) Jogos e novas tecnologias na educação. Perspectivas em Ciências Tecnológicas, v. 2, n. 2, p.39-48.

Barros Júnior, E. B. (2016) "Flat design em aplicativos móveis: definição, aplicações e avaliação de usabilidade". Monografia (Trabalho de Conclusão de Curso). UFPE, CAA, Design.

Birnbaum, A. (1957) "Efficient design and use of tests of mental ability for various decision-making problems". School of Aviation Medicine, Series Report No. 58-16, Washington DC: USAF School of Aviation Medicine.

Bocchini, B. (2018) "Pesquisa mostra evasão de 30\% em cursos superiores privados". AgênciaBrasilSãoPaulo.Educação.27/09/2018.Disponível em: http://agenciabrasil.ebc.com.br/educacao/noticia/2018-09/pesquisa-mostra-evasaode-30-em-cursos-superiores-privados

Brasil. (2011) Ministério da Educação. Instituto Nacional de Estudos e Pesquisas Educacionais Anísio Teixeira. “O que é TRI?" Junho de 2011. Disponível em: http://portal.inep.gov.br/artigo/-/asset_publisher/B4AQV9zFY7Bv/content/o-que-e$\operatorname{tri} / 21206$

Cristofolini, M., Oliari, D. E. (2015) "A Tendência Do Flat Design Usada Atualmente Na Identidade Visual Das Marcas". In: Intercom-XVI Congresso de Ciências da Comunicação na Região Sul. Joinville.

Embretson, S. E., Reise, S. P.(2000) "Item response theory for psychologists". Mahwah, NJ: L.

INEP. (2012) "Entenda a sua nota do ENEM Guia do participante”. Brasília-DF.

Machado, L. S., Costa, T. K. L., Moraes, R. M. (2018) Multidisciplinaridade e o desenvolvimento de serious games e simuladores para educaçao em saúde. Revista Observatório, v. 4, p. 149-172.

Mattar, J. (2010) “Games em educação: como os nativos digitais aprendem”. São Paulo: Pearson Prentice Hall.

Michael, D., David, R., Chen, S. L. (2005) "Serious games: Games that educate, train, and inform". Muska \& Lipman/Premier-Trade.

Neves, C. E. B, Raizer, L., Fachinetto, R. (2007) Educação superior para todos? Acesso, expansão e equidade: novos desafios para a política educacional. Sociologias, Porto Alegre, v.9, p. 124-157. 
O'Brien, H. L., Toms, E. G. (2008) What is user engagement? A conceptual framework for defining user engagement with technology. Journal of the American society for Information Science and Technology, v.59, p. 938-955.

Pasquali, L. (2007) “Teoria de Resposta ao Item - TRI”. Brasília: LabPAM/UnB.

Ratan, R., Rabindra, A., Ritterfeld, U. (2009) "Classifying serious games”. In: Serious games. Rougledge, p. 32-46.

Santos, G., Silva, L. A. (2011) "A evasão na educação superior entre o debate social e o objeto de pesquisa". In: Sampaio, S. (Org.). Observatório da vida estudantil: primeiros estudos. Salvador: EDUFBA.

Schell, J. (2011) “A arte de game design: o livro original”. Tradução: Edson Furmankiewicz. Rio de Janeiro: Elsevier.

Sena, A., Coelho, D. (2012) "Gameficação: uma análise das técnicas de engajamento atualmente utilizadas". SBC-Proceedings of SBGames.

Silva, A. C. S., Falcão, T. P. (2019) "Virtualização de Jogos Matemáticos: uma Avaliação do Cubra Doze". In Congresso Sobre Tecnologias Na Educação (CTRL+E) p. 50-59.

Tesser, G. J. (1994) Principais linhas epistemológicas contemporâneas. Educ. rev. n.10 Curitiba. Universidade Federal do Paraná.

Turkle, S. (1997) “A vida no ecrã: a identidade na era da Internet”. Lisboa: Relógio D’água.

Valente, J. A., Martins, M. C. (2011) O programa um computador por aluno e aformação de professores das escolas vinculadas à Unicamp. Revista GEMInIS, v. 2, p. 116-136.

Zichermann, G., Cunningham, C. (2011) "Gamification by Design: Implementing Game Mechanics in Web and Mobile Apps" 1st ed., Sebastopol (CAN): O'Reilly Media, Inc. 